TITLE:

Response of microscale turbulence and transport to the evolution of resistive magnetohydrodynamic magnetic island

$\operatorname{AUTHOR}(\mathrm{S}):$

Li, Jiquan; Kishimoto, Y.; Wang, Z. X.

CITATION:

Li, Jiquan ... [et al]. Response of microscale turbulence and transport to the evolution of resistive magnetohydrodynamic magnetic island. Physics of Plasmas 2014, 21(2): 020703.

ISSUE DATE:

2014-02

URL:

http://hdl.handle.net/2433/185158

RIGHT:

(C) 2014 AIP Publishing LLC 


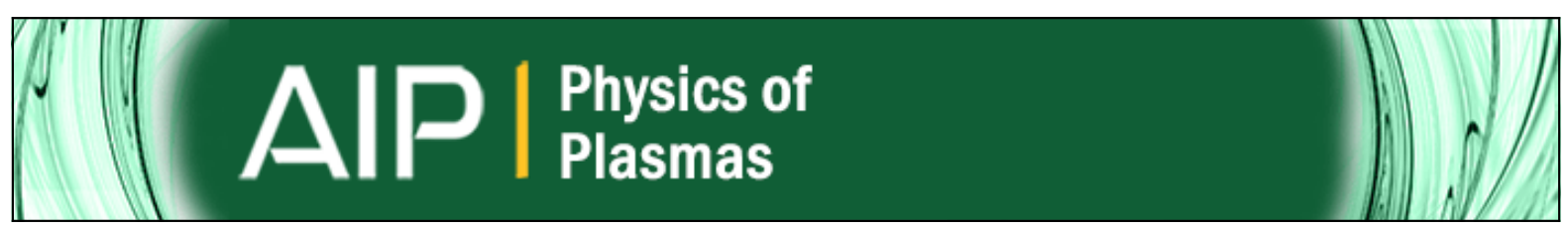

\title{
Response of microscale turbulence and transport to the evolution of resistive magnetohydrodynamic magnetic island
}

\author{
Jiquan Li, Y. Kishimoto, and Z. X. Wang
}

Citation: Physics of Plasmas (1994-present) 21, 020703 (2014); doi: 10.1063/1.4865378

View online: http://dx.doi.org/10.1063/1.4865378

View Table of Contents: http://scitation.aip.org/content/aip/journal/pop/21/2?ver=pdfcov

Published by the AIP Publishing

\section{Articles you may be interested in}

Suppressing electron turbulence and triggering internal transport barriers with reversed magnetic shear in the National Spherical Torus Experimenta)

Phys. Plasmas 19, 056120 (2012); 10.1063/1.4718456

Gyrokinetic simulations of mesoscale energetic particle-driven Alfvénic turbulent transport embedded in microturbulence

Phys. Plasmas 17, 112319 (2010); 10.1063/1.3509106

The effect of safety factor and magnetic shear on turbulent transport in nonlinear gyrokinetic simulations

Phys. Plasmas 13, 022305 (2006); 10.1063/1.2169804

Onset of intermittent thermal transport by ion-temperature-gradient-driven turbulence based on a low-degree-offreedom model

Phys. Plasmas 11, 3561 (2004); 10.1063/1.1751175

Zonal flows and transport in ion temperature gradient turbulence

Phys. Plasmas 9, 4911 (2002); 10.1063/1.1523010

\section{AlP Re-register for Table of Content Alerts}




\title{
Response of microscale turbulence and transport to the evolution of resistive magnetohydrodynamic magnetic island
}

\author{
Jiquan $\mathrm{Li}^{1,}{ }^{1, \mathrm{a})} \mathrm{Y}$. Kishimoto, ${ }^{1}$ and Z. X. Wang ${ }^{2}$ \\ ${ }^{1}$ Graduate School of Energy Science, Kyoto University, Uji, Kyoto 611-0011, Japan \\ ${ }^{2}$ School of Physics and Optoelectronic Technology, Dalian University of Technology, Dalian 116024, China
}

(Received 1 December 2013; accepted 30 January 2014; published online 11 February 2014)

Nonlinear evolution of microscale turbulence interacting with a naturally growing MHD magnetic island is simulated based on a Landau-fluid model. Here, we report on a new short wavelength magnetic-island-induced ion temperature gradient (ITG) instability triggered by a critical threshold of magnetic island width in multiscale turbulence, which is referred to as $s w-M I T G$ mode. The sw-MITG mode is characterized by a substantially low stability threshold and a global structure propagating along the ion diamagnetic drift direction. Its generation results from the response of microscale fluctuations to turbulent cross-field heat transport associated with increasing boundary layer width about the island separatrix. An intermittency of heat transport is caused by the sw-MITG mode interacting with dynamical magnetic island and microturbulence. (C) 2014 AIP Publishing LLC. [http://dx.doi.org/10.1063/1.4865378]

The dynamics of the magnetic island associated with magnetic reconnection is constantly a topic of intense research in laboratory and astrophysical plasmas. ${ }^{1-4}$ As a typical MHD phenomenon, island evolution through a tearing mode relies on MHD theory, in which the effect of microscale turbulence is modeled implicitly using anomalous resistivity or viscosity. ${ }^{5,6}$ However, the assumption on the scale separation may become questionable for the turbulent fluctuations with scale of the island width. On the other hand, the magnetic reconnection occurs, likely modifying the equilibrium for plasma stability. Furthermore, large parallel transport can flatten the pressure profile within the island, generally resulting in reduction of the microinstability drives. ${ }^{7,8}$ However, some exceptions with a peaking temperature or density profile inside the island separatrix have been diagnosed in some tokamak discharges. ${ }^{9,10}$ Accordingly, the microturbulence theory, such as the ion temperature gradient (ITG) turbulence with zonal flow dynamics ${ }^{11}$ with magnetic islands needs to be reevaluated, particularly for a large island. ${ }^{11}$

An important open issue relevant to the concerns above is the property of the microinstabilities and their nonlinear dynamics in MHD equilibrium with a magnetic island, particularly with a relatively slowly-developing or dynamical island. ${ }^{1}$ Theoretically, various scale fluctuations due to different instabilities may coexist and interplay with each other. Recently, several attempts have been made employing simplified gyrokinetic/fluid models or direct simulation. ${ }^{12-19}$ Most of them have analyzed the response of microscopic fluctuations and transport to a static island as a perturbation. The magnetic island is artificially embedded in a non-uniform plasma as a trial of the idea. The response of microscale fluctuations to a dynamical island is calling for consistent multiscale turbulence simulation. ${ }^{13,16,17}$ Note that complex multiscale interaction may cause a state transition due to secondary dynamic processes. Muraglia et al. ${ }^{16}$ observed a change of the final quasi-equilibrium state by the generation of small

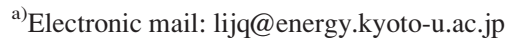

scale pressure structure due to the competition between the interchange and tearing modes. On the other hand, Ishizawa and Nakajima ${ }^{13}$ showed a secondary macro-MHD mode resulting from the multi-scale interaction between microturbulence and zonal flows in a quasi-steady equilibrium. Such nonlinear process may probably result from the state transition of the drift-tearing magnetic island equilibria for different diamagnetic drift frequency, predicted by Ottaviani et al. ${ }^{7}$

This Letter reports on a new short wavelength microinstability triggered by a critical magnetic island width in multiscale turbulence with a naturally growing MHD magnetic island. The new instability emerges in an island-dominated turbulent state after the flattening of ion temperature profile (FiTP) inside the island separatrix sharply vanishes while the flattening of density profile persists. This novel instability is regarded as a secondary nonlinear excitation, which offers new insights in understanding the complexity of multiscale multimode problem.

Simulations are carried out using a 5-field slab electromagnetic Landau-fluid model to focus on the underlying mechanism, which is composed of equations of normalized density $n$, vorticity $\nabla_{\perp}^{2} \phi$, magnetic flux $\psi$, parallel ion velocity $v$, and ion temperature $T_{i}{ }^{17}$

$$
\begin{gathered}
d_{t} n=-\partial_{y} \phi-\nabla_{/ /}(v-j)+D \nabla_{\perp}^{2} n, \\
d_{t} \nabla_{\perp}^{2} \phi=K \partial_{y} \nabla_{\perp}^{2} \phi+\nabla_{/ /} j+\mu \nabla_{\perp}^{4} \phi, \\
\beta \partial_{t} \psi=\nabla_{/ /}(\phi-n)-\beta \partial_{y} \psi+\eta j, \\
d_{t} v=-\nabla_{/ /}\left(2 n+T_{i}\right)-\beta(1+K) \partial_{y} \psi+\kappa \nabla_{\perp}^{2} v, \\
d_{t} T_{i}=-\eta_{i} \partial_{y} \phi-\frac{2}{3} \nabla_{/ / v}-\sqrt{\frac{32}{9 \pi}}\left|\nabla_{/ /}\right| T_{i}+\chi \nabla_{\perp}^{2} T_{i},
\end{gathered}
$$

with $d_{t}=\partial_{t}+\hat{e}_{z} \times \nabla_{\perp} \phi \cdot \nabla_{\perp}, \quad \nabla_{/ /}=\partial_{z}+\hat{s} \lambda \operatorname{arcsinh}(x / \lambda)$ $\partial_{y}-\hat{e}_{z} \times \nabla \psi \cdot \nabla_{\perp}, \nabla_{\perp}^{2}=\partial_{x}^{2}+\partial_{y}^{2}, j_{/ /}=\nabla_{\perp}^{2} \psi, \beta=8 \pi n_{0} T_{i 0} /$ 
$B^{2}, K=1+\eta_{i}$, and $\eta_{\mathrm{i}}=\partial \ln \mathrm{T}_{\mathrm{i} 0} / \partial \ln \mathrm{n}_{0}$. Here, $x$ is the distance deviated from the rational surface, $\hat{s}$ measures the local magnetic shear, and $\lambda$ denotes the gradient length of axial equilibrium current. Such an initial equilibrium corresponds to a given $q$ profile near the rational surface in tokamak plasmas. Here, the Landau-fluid closure ${ }^{20}$ is employed. In addition, classical cross-field dissipations $(D, \mu, \kappa, \chi)$ are also used at short scales.

Linear instability analysis ${ }^{17}$ shows the coexistence of both resistive tearing mode and ITG instability, which are individually governed by $\eta_{i}$ and the resistivity $\eta$. A 2D initial value code is employed to solve Eqs. (1)-(5) using Fourier decomposition in the $y$ direction with wave number $k_{y}=$ $2 \pi m / L_{y}$ and an implicit finite difference scheme in the $x$ direction with fixed boundary conditions at $x= \pm \mathrm{L}_{\mathrm{x}}$. In this work, $L_{x}=50$ is taken, which is much larger than the singular layer width. $L_{y}=20 \pi$ is taken so that the longest wavelength component $k_{y}=0.1$ corresponds to the unstable $m=1$ component of the tearing mode in a toroidal plasma. A reference parameter setting is fixed with $\beta=0.01$, $\hat{s}=0.2, \lambda=5 / \pi$, and $D=\mu=\kappa=\chi=0.01$ except for the key parameter pair $\left(\eta_{i}, \eta\right)$.

To explore the microturbulence behavior in a turbulent environment with naturally growing magnetic island, likely in the Rutherford stage of nonlinear tearing mode, simulations are performed by initially including both unstable tearing and ITG modes as a multiscale multimode scheme through adjusting $\eta_{i}$ and $\eta$. As a common feature, two instabilities grow independently at early linear phase. Afterwards, the stronger instability accelerates nonlinearly the growth of the weaker one. It is then saturated at a lower level due to the multiscale interaction, as shown in Fig. 1(a) by the evolution of kinetic energy. The mixed ITG-MHD fluctuations

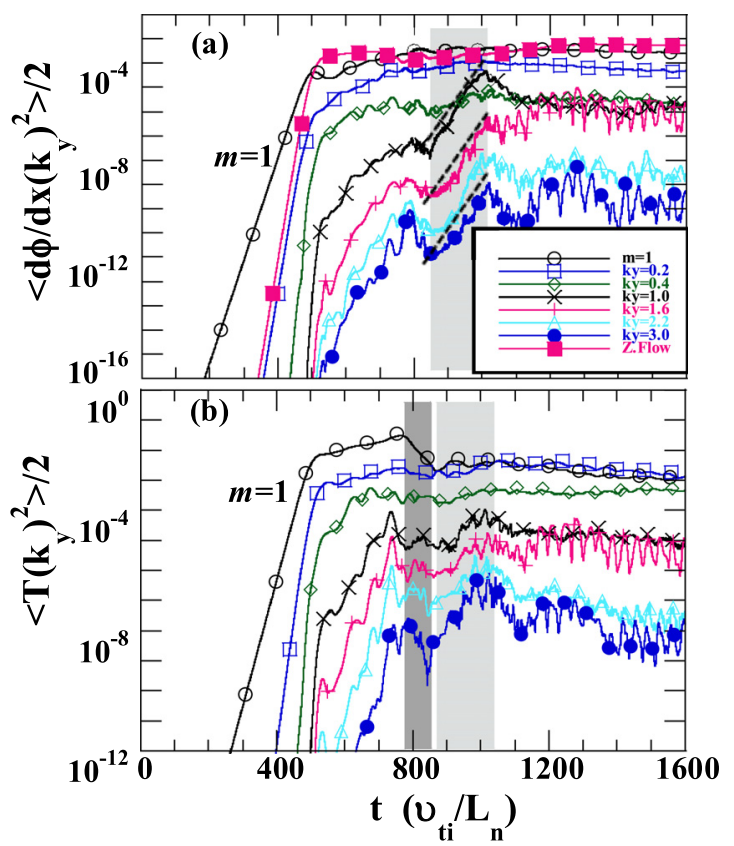

FIG. 1. Evolution of averaged kinetic energy (a) and fluctuating ion temperature (b). Here, $\eta_{i}=0.95, \eta=0.001$. The light and dark grey-shaded parts mark the phases of the sw-MITG excitation and the FiTP vanishing, respectively. Parallel dashed lines are for reference to measure averagely the growth rate of the sw-MITG mode. including the island evolution further grow after the first saturation. The spectral peak generally shifts to the MHD component regardless of the dominant ITG or tearing instability. Finally, the mixed turbulence is saturated again into a quasisteady-state dominated by a locked magnetic island. It is mention-worthy that all zonal components are involved here so that the quasi-linear profile relaxation, flow stabilization, and also island evolution/reconnection can take place consistently.

A pronounced observation in simulations shows a sudden growth of the short wavelength electrostatic potential in the quasi-steady-state, as shown by the grey-shaded interval in Fig. 1(a). Meanwhile, the magnetic island is still growing very gradually. The corresponding magnetic fluctuations are quite weak, showing an electrostatic character. All short wavelength components are of almost the same growth rate to form a global structure due to the mode coupling through the magnetic island. High resolution animations exhibit that the new instability propagates along the ion diamagnetic drift direction surrounding the island separatrix, opposed to the island rotation. These features look similar to those of the magnetic-island-induced ITG mode (MITG), which was predicted through a linear analysis under a presumption of uniform ion temperature gradient cross a static island. ${ }^{18}$ However, the phenomenon here occurs in the Rutherford regime due to the growing island, showing a state transition to the regime of high diamagnetic drift frequency. ${ }^{7}$ Hereafter, this new short wavelength instability is referred to as $s w$ MITG mode. Most importantly, it is observed that the FiTP inside the island separatrix sharply vanishes before the emergence of the sw-MITG mode. This evidence is symbolized by a sharp decrease of $m=1$ component of the ion temperature fluctuation, as shown in Fig. 1(b). It is also explicitly demonstrated by the change and contrast of reconstructed $T_{i}$ profiles at X-point and O-point before and after the FiTP vanishing, as shown in Fig. 2. Here, $T_{i}$ is reconstructed as the sum of all perturbation components and assumed equilibrium profile with local gradient, i.e., $T_{i}=\tilde{T}_{i}(x, y)-0.75 x$. Meanwhile, the flat density profile inside the island separatrix persists. The contour plots of the magnetic flux, ion temperature, and density profiles before and after the phase of the FiTP vanishing are displayed in Fig. 3. The sequence of the events shows that the FiTP vanishing is a precondition of the secondary excitation of the sw-MITG mode.

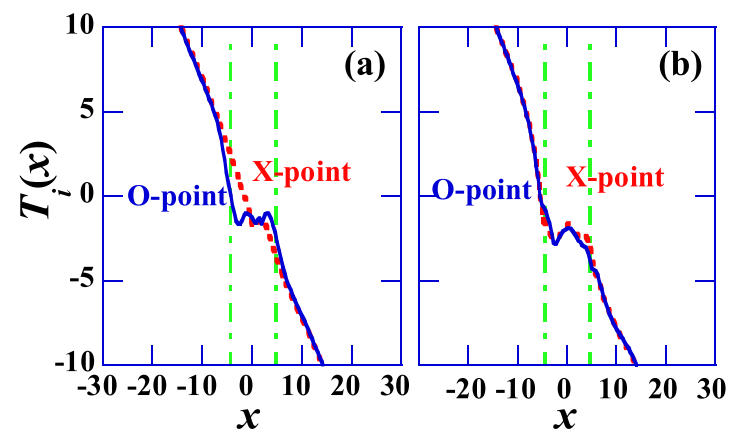

FIG. 2. Radial profiles of reconstructed $T_{i}$ at X-point and O-point at $\mathrm{t}=690$ (a) and $t=850$ (b) in the simulation of Fig. 1. The dotted-dashed lines mark the magnetic island width. 

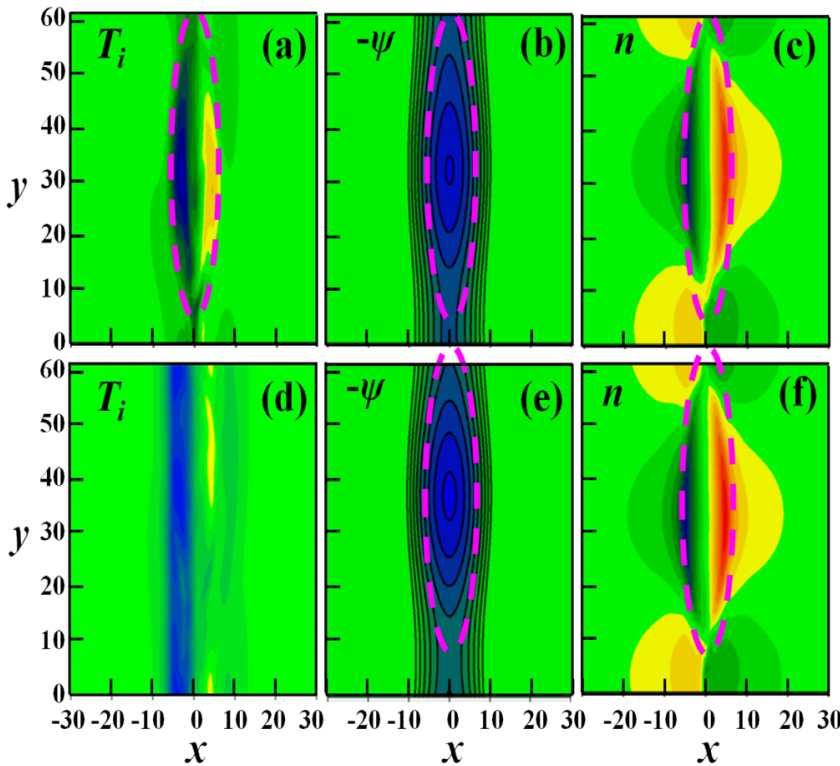

FIG. 3. Contour plots of $T_{i},-\psi$, and $n$ before ((a)-(c), $\left.t=690\right)$ and after ((d)-(f), $t=850)$ the FiTP vanishing in Fig. 1. The dashed curves indicate the magnetic island separatrix.

To quantify the features of the sw-MITG mode, the spectral characteristics are analyzed. Fig. 4(a) plots the evolution of $k_{y}$ spectra in the simulation of Fig. 1. A large spectral hump emerges around $k_{y} \rho_{i} \geq 1$ during and after the sw-MITG onset, which deviates from usual ITG spectrum of $k_{y} \rho_{i} \sim 0.4$. The saturation levels of both usual ITG mode at long wavelengths of $k_{y} \rho_{i} \sim 0.4$ and the sw-MITG mode around $k_{y} \rho_{i} \geq 1$ weakly depend on the initial $\eta_{i}$, as shown in Fig. 4(b). A parametric scan of growth rates of the MHD tearing, usual ITG and sw-MITG modes on $\eta_{i}$ is performed as shown in Fig. 5. The growth rates of both the tearing and usual ITG modes are directly measured in the early linear phase in nonlinear simulations for given $\eta_{i}$. Meanwhile, the growth rate of the sw-MITG instability is estimated averagely during the secondary excitation. It is shown that while usual $\eta_{i}$ dependences of both linear ITG and tearing modes are well reproduced (for the latter one, it should be almost independent of $\eta_{i}$ ), the sw-MITG mode is characterized by a substantially lower stability threshold. However, the dependence is not monotonically proportional to $\eta_{i}$. A large hump of growth rate appears for $\eta_{i} \leq 0.85$. For such weak ITG

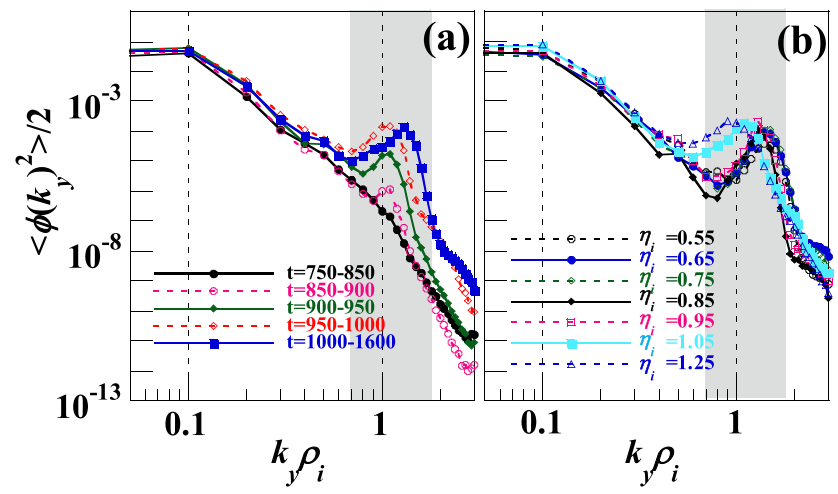

FIG. 4. (a) Evolution of averaged $k_{y}$ spectra in Fig. 1; (b) averaged $k_{y}$ spectra for different $\eta_{i}$. Here, $\eta=0.001$.

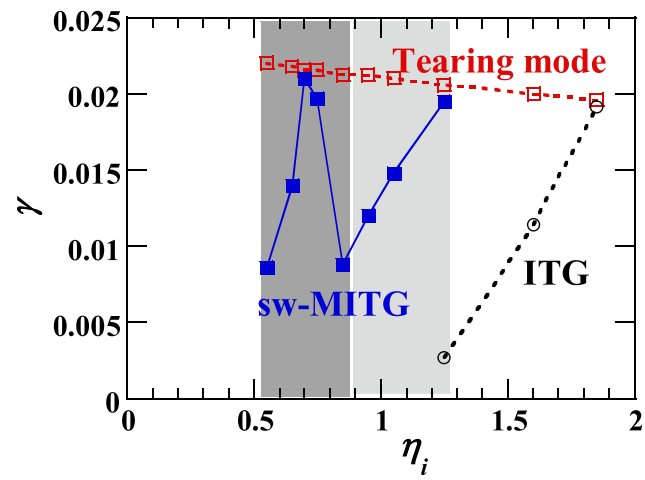

FIG. 5. Dependence of growth rates of the tearing, usual ITG and sw-MITG modes on $\eta_{i}$. Here, $\eta=0.001$.

drive, the simulations exhibit that the magnetic island continuously grows very gradually after the FiTP vanishes until the sw-MITG onset. This observation may suggest a critical threshold of the magnetic island width.

Motivated by the sequence of events and the stability analysis above, the magnetic island dynamics is investigated. Note that it is difficult to precisely measure the island width, $w$, in multiscale turbulence, an alternative estimation $w^{4} \propto$ $\left\langle\psi^{2}\right\rangle / 2$ may be applicable based on the relation $w^{2} \propto \psi{ }^{2}$ The averaged island widths are plotted in Fig. 6(a), in which the arrows mark the time of the sw-MITG onset for $\eta_{i} \leq 0.85$. An approximately, identical threshold of island width $w^{4} \propto$ $\left\langle\psi^{2}\right\rangle / 2 \sim 0.1$ is observed for the sw-MITG onset that is independent of $\eta_{i}$, showing an island-dominated regime, also as illustrated in Fig. 6(b). The actual threshold of island width is measured as $\sim 12 \rho_{\mathrm{i}}$ from Fig. 3. The time sequence of the events involving the FiTP vanishing, magnetic island growing,
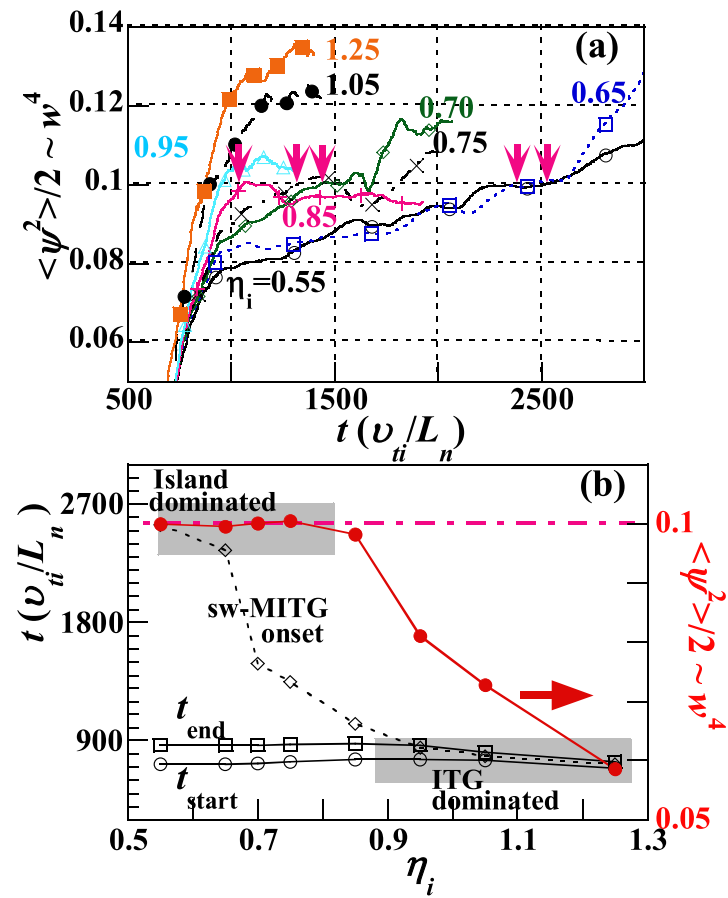

FIG. 6. (a) Evolution of the magnetic energy for different $\eta_{i}$. (b) The left vertical axis is the time sequence to start $\left(t_{\text {start }}\right)$ and end $\left(t_{\text {end }}\right)$ the FiTP vanishing, then excite the sw-MITG mode for different $\eta_{i}$. The right one corresponds to the critical island width for the sw-MITG onset versus $\eta_{i}$. Here, $\eta=0.001$. 

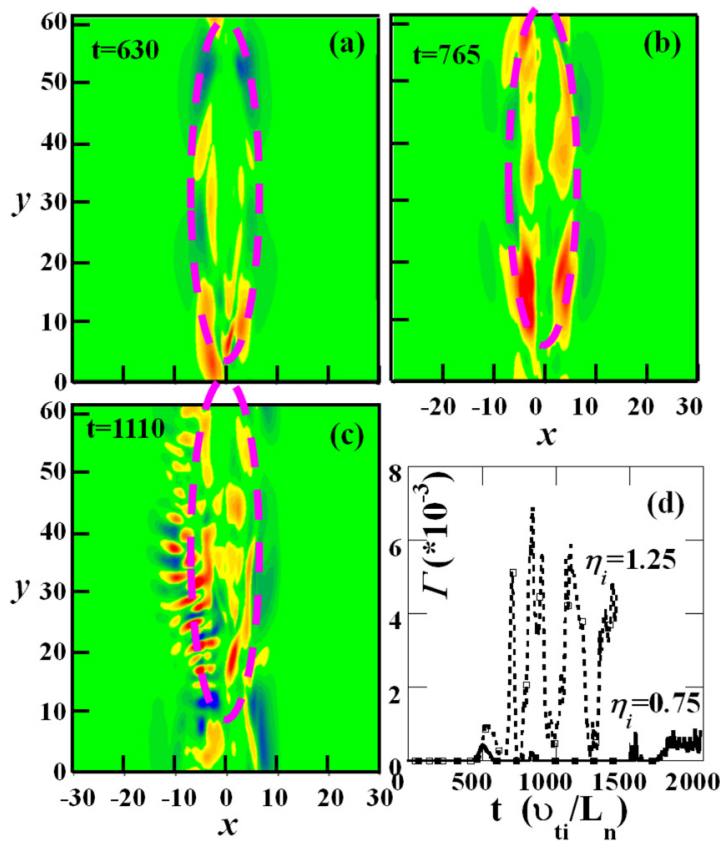

FIG. 7. (a)-(c) Contour plots of the heat flux before, during and after the FiTP vanishing Fig. 1. The dashed curves indicate the magnetic island separatrix. (d) Evolution of the heat flux $\Gamma=\left\langle\mathrm{T}_{\mathrm{i}} \partial_{\mathrm{y}} \phi\right\rangle$ for different $\eta_{i}$. Here, $\eta=0.001$

and sw-MITG onset in simulations as shown in Fig. 6(b) demonstrates two regimes for the sw-MITG excitation. One is dominated by the island width for $\eta_{i} \leq 0.85$, while the other is by the ITG for $\eta_{i}>0.95$. They correspond to the different $\eta_{i}$ dependence of the sw-MITG growth rates observed in Fig. 5. In the island-dominated regime with small $\eta_{i}$, the sw-MITG onset lags behind the FiTP vanishing with a long waiting time for the island growing to the critical threshold width. On the other hand, the sw-MITG onset in the ITG-dominated regime occurs during the phase of the FiTP vanishing as illustrated by the dashed curve in Fig. 6(b), showing a synergetic destabilization of both ITG and magnetic island width. ${ }^{18}$

The FiTP vanishing is a key factor for the sw-MITG excitation. Here, the ion heat transport near the island separatrix is analyzed. Note that the FiTP within an island depends on the boundary layer compared to the island width through the energy balance $\chi_{/ /} \nabla_{/ /}^{2} \mathrm{~T}+\chi_{\perp} \nabla_{\perp}^{2} \mathrm{~T}=0$, where $\chi_{/ /}$and $\chi_{\perp}$ are the parallel and perpendicular thermal conductivities, respectively. ${ }^{1} \mathrm{~A}$ critical island width determining the FiTP is predicted as $w_{C} \propto a\left(\chi_{\perp} / \chi_{/ /}\right)^{1 / 4}$ below which the FiTP tends to vanish. ${ }^{21,22}$ The cross-field transport produced by the microturbulence enhances $\chi_{\perp}$ so that $w_{C}$ may be influenced by the ITG modes. In the ITG-dominated regime, larger $\chi_{\perp}$ due to the ITG turbulence associated with the steepened temperature gradient in the boundary layer by the zonal temperature may increase $w_{C}$ faster than the island growth. As a result, the dynamical island width becomes lower than $w_{C}$, leading to the FiTP vanishing. To examine such a mechanism, the evolution of heat flux about the island separatrix is evaluated in the phases before, during and after the FiTP vanishing, as shown in Figs. 7(a)-7(c). The boundary layer of heat flux evidently becomes wider during the FiTP vanishing, showing a spontaneous breakdown of FiTP across the island. Finally, the sw-MITG mode also enhances the cross-field heat transport to cause a bursty transport behavior, as illustrated in Fig. 7(d). Such intermittency results from the multiscale multimode interaction dynamics among the tearing mode including the magnetic island, the usual long wavelength ITG mode and the secondary sw-MITG mode, which is further discussed in more detail elsewhere.

In summary, we showed a novel short wavelength microscale ITG instability with substantially lower stability threshold and global mode structure, which is triggered by a critical threshold of the magnetic island width. This secondary instability results from the response of micro-scale fluctuations to both the growing island and the turbulent cross-field heat transport about the island separatrix. It may correspond to a state transition of the drift-tearing magnetic island equilibria for different diamagnetic drift frequency. ${ }^{7}$ The resultant heat transport behaves with an intermittency due to the multiscale interaction between both the MHD and ITG modes involving the new sw-MITG instability. Similar phenomenon was observed in natural plasmas, in which nonlinear growth of high mode number ballooning instability in a thin current sheet may lead to the plasmoid formation in the high Lundquist number magnetotail. ${ }^{23,24}$

${ }^{1}$ P. H. Rutherford, Phys. Fluids 16, 1903 (1973).

${ }^{2}$ A. I. Smolyakov, Plasma Phys. Controlled Fusion 35, 657 (1993).

${ }^{3}$ M. Yamada, Phys. Plasmas 14, 058102 (2007).

${ }^{4}$ F. L. Waelbroeck, Nucl. Fusion 49, 104025 (2009).

${ }^{5}$ K. Itoh, S.-I. Itoh, and A. Fukuyama, Phys. Rev. Lett. 69, 1050 (1992).

${ }^{6}$ P. Diamond, R. D. Hazeltine, Z. G. An, B. A. Carreras, and H. R. Hicks, Phys. Fluids 27, 1449 (1984)

${ }^{7}$ M. Ottaviani, F. Porcelli, and D. Grasso, Phys. Rev. Lett. 93, 075001 (2004).

${ }^{8}$ B. Scott, A. B. Hassam, and J. F. Drake, Phys. Fluids 28, 275 (1985).

${ }^{9}$ P. C. de Vries, A. J. H. Donne, S. H. Heijnen, C. A. J. Hugenholtz, A. Kramer-Flecken, F. C. Schuller, and G. Waidmann, Nucl. Fusion 37, 1641 (1997).

${ }^{10}$ K. Ida, K. Kamiya, A. Isayama, Y. Sakamoto, and JT-60 Team, Phys. Rev. Lett. 109, 065001 (2012)

${ }^{11}$ W. Horton, Rev. Mod. Phys. 71, 735 (1999), and references therein.

${ }^{12}$ C. J. McDevitt and P. H. Diamond, Phys. Plasmas 13, 032302 (2006).

${ }^{13}$ A. Ishizawa and N. Nakajima, Phys. Plasmas 14, 040702 (2007).

${ }^{14}$ F. L. Waelbroeck, F. Militello, R. Fitzpatrick, and W. Horton, Plasma Phys. Controlled Fusion 51, 015015 (2009).

${ }^{15}$ H. R. Wilson and J. W. Connor, Plasma Phys. Controlled Fusion 51, 115007 (2009)

${ }^{16}$ M. Muraglia, O. Agullo, S. Benkadda, X. Garbet, P. Beyer, and A. Sen, Phys. Rev. Lett. 103, 145001 (2009).

${ }^{17}$ J. Li, Y. Kishimoto, Y. Kouduki, Z. X. Wang, and M. Janvier, Nucl. Fusion 49, 095007 (2009).

${ }^{18}$ Z. X. Wang, J. Q. Li, Y. Kishimoto, and J. Q. Dong, Phys. Plasmas 16, 060703 (2009).

${ }^{19}$ W. A. Hornsby, A. G. Peeters, M. Siccinio, and E. Poli, Phys. Plasmas 19, 032308 (2012).

${ }^{20}$ G. W. Hammett and P. W. Perkins, Phys. Rev. Lett. 64, 3019 (1990).

${ }^{21}$ R. Fitzpatrick, Phys. Plasmas 2, 825 (1995).

${ }^{22}$ R. D. Hazeltine, P. Helander, and P. J. Catto, Phys. Plasmas 4, 2920 (1997).

${ }^{23}$ A. Bhattacharjee, Z. W. Ma, and X. G. Wang, Geophys. Res. Lett. 25, 861, doi:10.1029/98GL00412 (1998).

${ }^{24}$ P. Zhu and J. Raeder, Phys. Rev. Lett. 110, 235005 (2013). 